\title{
Effects of Gadolinium Contrast Agent Administration on Automatic Brain Tissue Classification of Patients with Multiple Sclerosis
}

\author{
J.B.M. Warntjes, A. Tisell, A.-M. Landtblom, and P. Lundberg
}

\begin{abstract}
BACKGROUND AND PURPOSE: The administration of gadolinium contrast agent is a common part of MR imaging examinations in patients with MS. The presence of gadolinium may affect the outcome of automated tissue classification. The purpose of this study was to investigate the effects of the presence of gadolinium on the automatic segmentation in patients with MS by using the synthetic tissue-mapping method.
\end{abstract}

MATERIALS AND METHODS: A cohort of 20 patients with clinically definite multiple sclerosis were recruited, and the T1 and T2 relaxation times and proton density were simultaneously quantified before and after the administration of gadolinium. Synthetic tissue-mapping was used to measure white matter, gray matter, CSF, brain parenchymal, and intracranial volumes. For comparison, 20 matched controls were measured twice, without gadolinium.

RESULTS: No differences were observed for the control group between the 2 measurements. For the MS group, significant changes were observed pre- and post-gadolinium in intracranial volume $(-13 \mathrm{~mL}, P<.005)$ and cerebrospinal fluid volume $(-16 \mathrm{~mL}, P<.005)$ and the remaining, unclassified non-WM/GM/CSF tissue volume within the intracranial volume $(+8 \mathrm{~mL}, P<.05)$. The changes in the patient group were much smaller than the differences, compared with the controls, which were $-129 \mathrm{~mL}$ for WM volume, $-22 \mathrm{~mL}$ for GM volume, $+91 \mathrm{~mL}$ for CSF volume, $24 \mathrm{~mL}$ for the remaining, unclassified non-WM/GM/CSF tissue volume within the intracranial volume, and $-126 \mathrm{~mL}$ for brain parenchymal volume. No significant differences were observed for linear regression values against age and Expanded Disability Status Scale.

CONCLUSIONS: The administration of gadolinium contrast agent had a significant effect on automatic brain-tissue classification in patients with MS by using synthetic tissue-mapping. The observed differences, however, were much smaller than the group differences between MS and controls.

ABBREVIATIONS: $B P V=$ brain parenchymal volume; $\mathrm{CSFV}=$ cerebrospinal fluid volume; $\mathrm{EDSS}=$ Expanded Disability Status Scale; $\mathrm{Gd}=$ gadolinium; $\mathrm{GMV}=$ gray matter volume; ICV = intracranial volume; NV = the remaining, unclassified non-WM/GM/CSF tissue volume within the ICV, defined as ICV - (WMV + GMV + CSFV); $\mathrm{PD}=$ proton density; $\mathrm{WMV}=$ white matter volume

M ultiple sclerosis is a chronic inflammatory disorder of the central nervous system. Typically, focal white matter lesions are regarded as a hallmark pathologic finding. These le-

Received October 9, 2013; accepted after revision December 13.

From the Center for Medical Image Science and Visualization, (J.B.M.W., A.T., P.L.) and Clinical Physiology (J.B.M.W.) and Radiation Physics (P.L.), Department of Medical and Health Sciences, Linköping University, Linköping, Sweden; Departments of Clinical Physiology (J.B.M.W.), Clinical Neuroscience (A.-M.L.), and Radiation Physics (P.L.), UHL, County Council of Östergötland, Linköping, Sweden; Radiation Physics (A.T.), Department of Medical and Health Sciences, Faculty of Health Sciences, Linköping University, Linköping, Sweden; and Neurology, Department of Clinical and Experimental Medicine (A.-M.L.), Linköping University, Linköping, Sweden.

This work was supported in part by Grant No. VR/NT 2008-3368 from the National Research Council.

Please address correspondence to J.B.M. Warntjes, Center for Medical Image Science and Visualization, Linköping University Hospital, Linköping 58185, Sweden; e-mail: marcel.warntjes@cmiv.liu.se

- Indicates open access to non-subscribers at www.ajnr.org

http://dx.doi.org/10.3174/ajnr.A3890 sions, however, show a limited correlation with clinical findings. ${ }^{1-3}$

Instead, brain-tissue loss shows a stronger correlation with disease progression, especially in a later stage of the disease. ${ }^{3-9}$ The key for the practical, clinical application of brain-tissue volume measurements is an automated, fast method that can be integrated into the clinical workflow. Numerous automated methods exist for this purpose, generally based on conventional T1weighted, T2-weighted, and FLAIR images or a combination thereof. In these methods, tissue is classified on signal-intensity differences in the images where each pixel can be assigned to 1 specific tissue type $\mathrm{e}^{10-15}$ or multiple tissue types such that partial volume effects are accounted for. ${ }^{16-19}$ The latter method is advantageous because it decreases the dependency on image resolution.

Despite the obvious benefits, brain volumetric measurements are not widely used in clinical practice. One of the main difficulties is that the image intensity of the input images can vary sub- 
stantially due to scanner configuration and examination parameters $^{20}$ and age- or disease-related tissue changes, ${ }^{21,22}$ leading to differences in classification. Second, extensive manual interaction of the postprocessing software or long processing times may be required, which may lead to hours or even days of lag time to retrieve the volumetric information. ${ }^{23}$ A complicating factor for automatic segmentation methods is the use of contrast agents. Gadolinium (Gd) contrast agent is generally administered to patients with MS to highlight any damage to the blood-brain barrier, indicating inflammatory lesions and helping to stage disease evolution. Leakage of Gd into brain tissue strongly reduces the $\mathrm{T} 1$ relaxation, resulting in hyperintensity on T1-weighted images. Even in the absence of leakage, the presence of Gd in the capillary network of the brain parenchyma may slightly reduce $\mathrm{T} 1$ relaxation of the brain tissue, which could have a secondary effect on automated tissue-classification methods. This effect may even vary with the amount and timing of contrast administration.

In this work, the synthetic tissue-mapping (SyMap) method, which previously reported on measurements of the intracranial volume (ICV), ${ }^{24}$ the brain parenchymal fraction, ${ }^{25}$ and all tissue fractions, ${ }^{16}$ was evaluated for brain-tissue measurements in patients with MS with and without the presence of a Gd contrast agent. The method is based on quantitative MR imaging to measure the longitudinal relaxation time, T1; the transverse relaxation time, T2; and proton density. ${ }^{26}$ The procedure does not generate conventional MR images but maps of physical parameters that directly reflect tissue properties. Such a quantitative measurement removes a large number of dependencies of MR imaging scanner settings and imperfections. ${ }^{27}$ The method includes a fully automatic postprocessing software, to calculate the white matter volume (WMV), gray matter volume (GMV), cerebrospinal fluid volume (CSFV), the remaining, unclassified non-WM/GM/CSF tissue volume within the ICV (NV), brain parenchymal volume (BPV), and ICV on the basis of the measured T1, T2, and PD maps. The total postprocessing time is $<1$ minute, which makes it appropriate for routine clinical purposes.

The aim of this study was to evaluate the use of synthetic tissue-mapping for the measurement of WMV, GMV, CSFV, NV, $\mathrm{BPV}$, and ICV in patients with MS pre- and post-Gd. In addition, the brain-tissue fractions of WM, GM, CSF, remaining, unclassified non-WM/GM/CSF tissue, and brain parenchyma, normalized against the ICV, were evaluated. The repeatability of the automatic brain segmentation was assessed by measuring the controls twice.

\section{MATERIALS AND METHODS \\ Subjects}

The study included a group of 20 patients ( 5 men, 15 women; mean age, $47 \pm 12$ years) diagnosed with clinically definite MS based on clinical presentation and laboratory findings. All patients fulfilled the Poser criteria with at least 2 relapses, separated in space and time. ${ }^{28,29}$ The mean Expanded Disability Status Scale (EDSS) score ${ }^{30}$ for patients was $3.8 \pm 2.3$ (median, 3.5; range, 1.0-7.5). EDSS is a method of evaluating the degree of neurologic impairment in MS, scoring 8 functional systems, resulting in steps of 0.5 from 0 (healthy) to 10 (death due to MS). The mean disease duration was $15 \pm 11$ years. No differentiation was made between types of MS, and the group consisted of 12 patients with relapsing- remitting and 8 with secondary-progressive MS. One patient was excluded from the analysis due to severe MR imaging receive coil signal-intensity problems. The study was part of routine follow-up examinations. For comparison, a group of 20 age- and sex-matched healthy controls was included ( 5 men, 15 women; mean age, $48 \pm 12$ years). The mean age difference compared with the MS group was $0.7 \pm 2.7$ years. The study was approved by the Regional Ethics Committee (reference number Dnr M88-07), and written informed consent was obtained from all participants.

\section{Scanning Protocol}

The MR imaging quantification method QRAPMASTER (also known as Qmap) ${ }^{26}$ was performed to simultaneously retrieve the $\mathrm{T} 1$ and $\mathrm{T} 2$ relaxation and proton density. The sequence was a multi-spin-echo saturation recovery sequence with 4 saturation delays and 5 echoes. Hence, the sequence produced a matrix of $4 \times 5=20$ images per slice with the combined effects of T1 and T2 relaxation in the image intensity. The saturation delay times were at $100,400,1380$, and $2860 \mathrm{~ms}$ with a TR of $2950 \mathrm{~ms}$. The TEs were 14, $28,42,56$, and $70 \mathrm{~ms}$. The in-plane resolution was $1 \times 1 \mathrm{~mm}^{2}$ over an FOV of $210 \mathrm{~mm}$; 30 axial sections of $4 \mathrm{~mm}$ thickness (no gap) were acquired in a scan time of 8 minutes and 21 seconds. The MR imaging scanner was an Achieva 1.5T (Philips Healthcare, Best, the Netherlands). All subjects were scanned twice with 20 minutes between the scans. Only the patient group received a single dose of gadopentetate dimeglumine contrast agent $(0.2 \mathrm{~mL} / \mathrm{kg}$, Magnevist $0.5 \mathrm{mmol} /$ $\mathrm{mL}$; Schering, Berlin, Germany) 10 minutes before the second acquisition.

\section{Image Postprocessing}

The raw data were analyzed with the SyMRI 7.0 software (Synthetic MR, Linköping, Sweden) to retrieve the T1, T2, and PD maps. These maps were used as input for the automatic brain segmentation in the same software. In summary, WM, GM, and CSF tissue clusters and mixtures thereof were recognized as specific combinations of T1, T2, and PD values, as previously reported. ${ }^{16}$ The total ICV comprised all recognized WM, GM, and CSF, for which a region-growing algorithm ensured a contiguous volume. The border of the ICV was refined to set the threshold at $\mathrm{PD}=50 \%$, by using the definition of the tissue interface between CSF (with visible $\mathrm{PD}=100 \%$ ) and bone (with visible $\mathrm{PD}=0 \%$ ). The ICV was automatically cut at the base of the skull. The sum of all WM, GM, and CSF partial volumes inside the ICV provided the WMV, GMV, and CSFV, respectively. The BPV was defined as the ICV minus the CSFV. The region-growing algorithm leads to the inclusion of volume that does not match the defined WM, $\mathrm{GM}$, or CSF characteristics within the ICV. This remaining tissue was labeled the non-WM/GM/CSF volume, comprising unspecified tissue such as blood vessels, motion artifacts, or pathologic tissue. Normalization with the ICV resulted in the white matter fraction, gray matter fraction, CSF fraction, the non-WM/GM/ CSF fraction, and the brain parenchymal fraction. The software did not have a standard brain or any atlas-driven models; tissue was segmented on T1-T2-PD characteristics only.

Loading all raw data from a PACS required 20-30 seconds, and calculating the T1, T2, and PD maps with subsequent seg- 


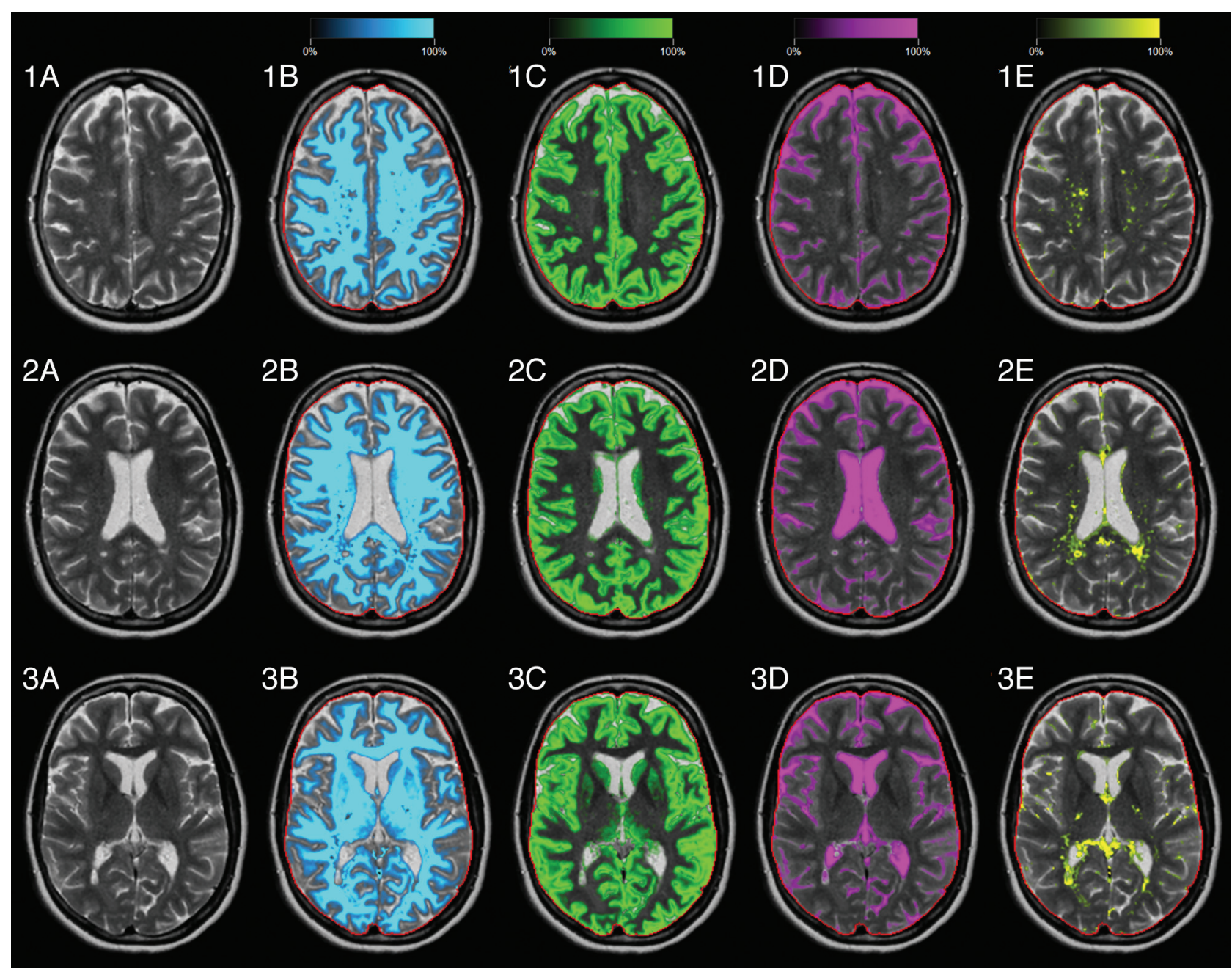

FIG 1. Typical images of the automatic segmentation software of a patient with MS (39-year-old woman, EDSS $=4.0)$. Three slices are shown, numbers 19,16 , and 13 of the 30 acquired slices. $A, T 2$-weighted image. $B$, White matter segmentation, in which the intensity of the light-blue color overlay corresponds to the calculated white matter partial volume per voxel. The red line indicates the intracranial volume. Similar images are shown for gray matter in green $(C)$, CSF in pink (D), and non-WM/GM/CSF in yellow $(E)$.

mentation of the brain was performed within 10-20 seconds. A standard 64-bit PC with 6 gigabytes of RAM was used.

\section{Statistics}

For the WMV, GMV, CSFV, NV, BPV, and ICV and for WM fraction, GM fraction, CSF fraction, remaining, unclassified nonWM/GM/CSF tissue fraction, and brain parenchymal fraction, the mean value and SD were calculated for each measurement. Linear regression was used to analyze the relation of the volume and fraction values to age. The results from the regression analysis of the controls were used to correct the values of the patients with MS for age. Linear regression with EDSS was then performed as a separate step. A Shapiro-Wilk test was applied for normal distribution. A paired $t$ test was performed to find the significance of the difference between measurement 1 and measurement 2. A 1-way ANOVA with post hoc Tukey analysis was used to assess group differences between controls and patients with MS.

\section{RESULTS}

Typical results of the segmentation software on 3 of the 30 acquired slices are shown in Fig 1. An axial slice of the head of one of the patients with MS (a 39-year-old woman; EDSS = 4.0) is displayed. In the left column, the T2-weighted images are shown for comparison. On the right, the calculated WM, GM, CSF, and non-WM/GM/CSF partial volume maps are displayed as a color overlay in which the color intensity corresponds to the partial volume in the range between $0 \%$ and $100 \%$ tissue. The red line indicates the border of the ICV. The mean ICV of all subjects at the first measurement was $1387 \mathrm{~mL}$, with an SD of $110 \mathrm{~mL}$ (7.9\%). White matter lesions mainly show up as the remaining non-WM/ GM/CSF but are partly recognized as GM and CSF. Radiologic inspection showed that no patient had Gd-enhancing lesions.

In Table 1, the results of the fully automatic tissue volume measurements are shown for WMV, GMV, CSFV, NV, BPV, and ICV. No statistically significant difference was found between the 2 measurements for all volumes of the control group. The differences for the patient group were much larger; a significant reduction of the ICV of $13 \mathrm{~mL}$ and of the CSFV of $16 \mathrm{~mL}$ was observed as patients were exposed to Gd. The differences in BPV, WM, and GM were not significant, but the ratio of WMV and GMV changed toward higher WMV. Visual inspection did not result in locating a specific area in which differences in volumes were pro- 
Table 1: Fully automatic measurements of the WMV, GMV, CSFV, NV, BPV, and ICV volumes of the control group and the MS group ${ }^{\text {a }}$

\begin{tabular}{lcccccc}
\hline & WMV (mL) & GMV (mL) & CSFV (mL) & NV (mL) & BPV (mL) & ICV (mL) \\
\hline Contr1 & $581 \pm 67$ & $639 \pm 58$ & $156 \pm 45$ & $28 \pm 7$ & $1247 \pm 119$ & $1404 \pm 119$ \\
Contr2 & $581 \pm 69$ & $637 \pm 53$ & $156 \pm 47$ & $31 \pm 12$ & $1250 \pm 119$ & $1406 \pm 120$ \\
MS1 & $452 \pm 88$ & $617 \pm 48$ & $247 \pm 60$ & $52 \pm 22$ & $1122 \pm 99$ & $1369 \pm 99$ \\
MS2 & $464 \pm 109$ & $600 \pm 47$ & $231 \pm 60$ & $60 \pm 21$ & $1124 \pm 98$ & $1355 \pm 97$ \\
Diff Contrl-Contr2 & $0 \pm 11$ & $-1 \pm 13$ & $0 \pm 4$ & $4 \pm 12$ & $2 \pm 6$ & $2 \pm 7$ \\
Diff MS1-MS2 & $12 \pm 37$ & $-17 \pm 40$ & $-16 \pm 7^{\mathrm{c}}$ & $8 \pm 11^{\mathrm{b}}$ & $2 \pm 9$ & $-13 \pm 8^{\mathrm{c}}$ \\
Diff Contrl-MS1 & $-129 \pm 104^{\mathrm{c}}$ & $-22 \pm 68$ & $91 \pm 71^{\mathrm{c}}$ & $24 \pm 18^{\mathrm{c}}$ & $-126 \pm 133^{\mathrm{c}}$ & $-35 \pm 117$ \\
Diff Contr2-MS2 & $-117 \pm 113^{\mathrm{c}}$ & $-37 \pm 67^{\mathrm{b}}$ & $75 \pm 70^{\mathrm{c}}$ & $29 \pm 17^{\mathrm{c}}$ & $-126 \pm 136^{\mathrm{c}}$ & $-50 \pm 116$ \\
\hline
\end{tabular}

Note:-Diff indicates difference; Contr, control group; MS, MS patient group; 1, measurement 1; 2, measurement 2.

${ }^{a}$ For each tissue volume, the mean value and SD are given. The mean difference and SD of the first measurement and the second measurement and between controls and patients with MS are also provided. The MS group received Gd in the second measurement.

b $P<.05$ (significant difference).

$c p<.005$ (significant difference).

Table 2: Fully automatic measurements of the normalized WMF, GMF, CSFF, NF, and BPF of the control group and the MS group ${ }^{\mathrm{a}}$

\begin{tabular}{lccccc}
\hline & WMF (\%) & GMF (\%) & CSFF (\%) & NF (\%) & BPF (\%) \\
\hline Contr1 & $41.4 \pm 2.6$ & $45.5 \pm 1.9$ & $11.2 \pm 3.1$ & $2.0 \pm 0.5$ & $88.8 \pm 3.1$ \\
Contr2 & $41.3 \pm 2.4$ & $45.4 \pm 1.8$ & $11.1 \pm 3.1$ & $2.2 \pm 0.9$ & $88.9 \pm 3.1$ \\
MS1 & $32.9 \pm 5.4$ & $45.2 \pm 3.0$ & $18.0 \pm 4.1$ & $3.8 \pm 1.7$ & $82.0 \pm 4.1$ \\
MS2 & $34.0 \pm 6.8$ & $44.5 \pm 4.6$ & $17.1 \pm 4.2$ & $4.4 \pm 1.6$ & $82.9 \pm 4.2$ \\
Diff Contrl-Contr2 & $-0.1 \pm 0.9$ & $-0.1 \pm 0.8$ & $0.0 \pm 0.3$ & $0.2 \pm 0.8$ & $0.0 \pm 0.3$ \\
Diff MS1-MS2 & $1.1 \pm 2.9$ & $-0.7 \pm 3.2$ & $-1.0 \pm 0.5^{c}$ & $0.6 \pm 0.8^{\mathrm{b}}$ & $1.0 \pm 0.5^{\mathrm{c}}$ \\
Diff Contrl-MS1 & $-8.4 \pm 6.1^{\mathrm{c}}$ & $-0.4 \pm 3.4$ & $6.9 \pm 5.1^{\mathrm{c}}$ & $1.9 \pm 1.5^{\mathrm{c}}$ & $-6.9 \pm 5.1^{\mathrm{C}}$ \\
Diff Contr2-MS2 & $-7.3 \pm 6.9^{\mathrm{c}}$ & $-0.9 \pm 4.3$ & $6.0 \pm 5.1^{\mathrm{c}}$ & $2.2 \pm 1.4^{\mathrm{c}}$ & $-6.0 \pm 5.1^{\mathrm{c}}$ \\
\hline
\end{tabular}

Note:-Diff indicates difference; Contr, control group; MS, MS patient group; 1, measurement 1; 2, measurement 2; WMF, WM fraction; GMF, GM fraction; CSFF, CSF fraction; NF, remaining, unclassified non-WM/GM/CSF tissue fraction; BPF, brain parenchymal fraction.

a Each tissue fraction corresponds to the tissue volume divided by the ICV. The mean value and SD are given as well as the mean difference and SD of the first measurement and the second measurement and between the controls and patients with MS.

${ }^{b} P<.05$ (significant difference)

${ }^{c} P<.005$ (significant difference).
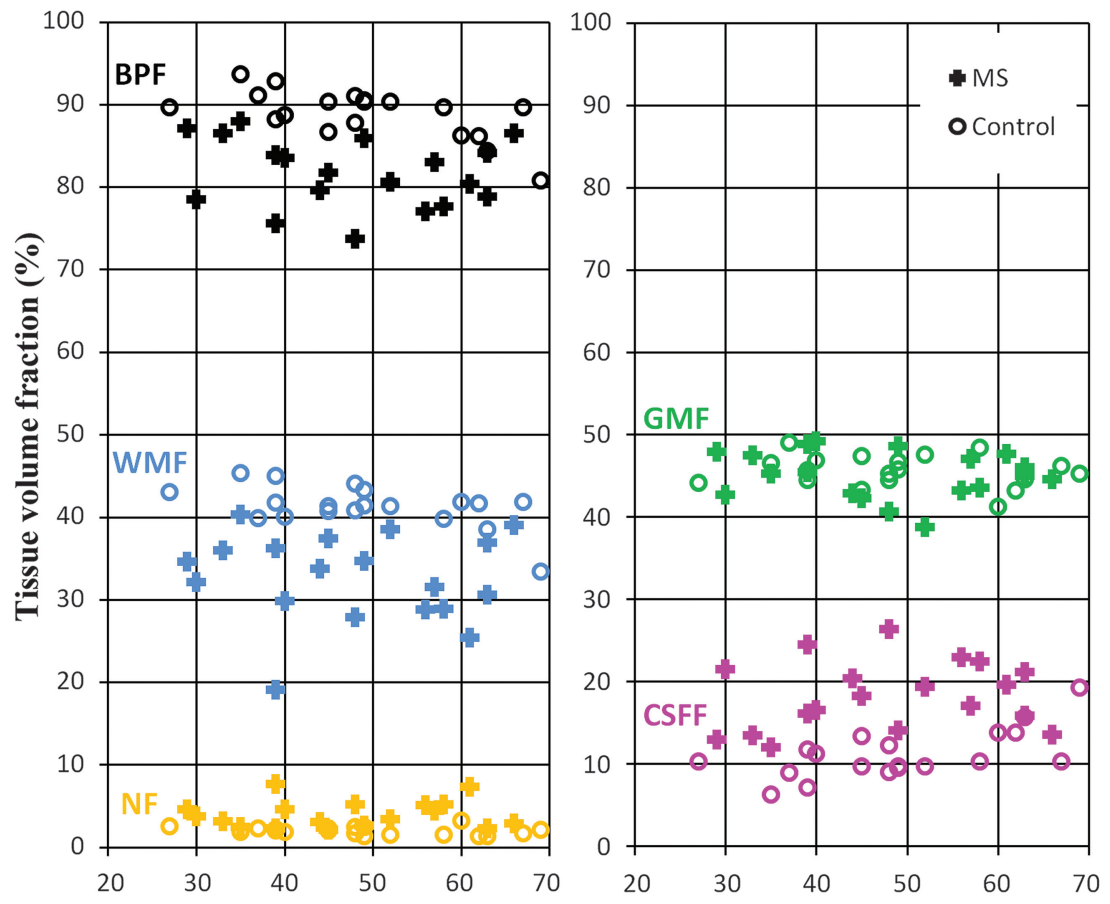

Subject age (years)

FIG 2. Brain-tissue fraction results of the first measurement: the brain parenchymal fraction, white matter fraction, gray matter fraction, CSF fraction, and the non-WM/GM/CSF fraction of the intracranial volume, as a function of subject age. Markers are zero for the control group and plus for the MS group. The colors are similar to the segmentation overlay colors of Fig 1 . nounced. The observed differences were distributed over all slices and ranged between 0 and $1 \mathrm{~mL}$ per section. Between the control and patient groups, large volume differences were observed. The mean BPV was $126 \mathrm{~mL}$ smaller for the MS group, similar to the total mean WMV difference $(129 \mathrm{~mL})$. The CSFV was significantly larger for patients, with a mean difference of $91 \mathrm{~mL}$. In addition, the remaining NV was larger $(24 \mathrm{~mL})$.

In Table 2, the normalized tissue fractions of WM, GM, CSF, remaining, unclassified non-WM/GM/CSF tissue, and brain parenchyma are shown. Again only small differences were observed for the control group. For the MS group, on the other hand, the WM fraction, remaining, unclassified non-WM/GM/CSF tissue fraction, and brain parenchymal fraction increased, whereas the GM fraction and CSF fraction decreased after Gd administration. The changes in CSF fraction, remaining, unclassified non-WM/GM/CSF tissue fraction, and brain parenchymal fraction were significant. When one compared the 2 groups, a significant difference in WM fraction, CSF fraction, remaining, unclassified non-WM/GM/CSF tissue fraction, and brain parenchymal fraction between the controls and the patients with MS was observed. The differences between the 2 groups are visualized in Fig 2, where the normalized WM fraction, GM fraction, CSF fraction, remaining, unclassified non-WM/GM/CSF tissue fraction, and brain parenchymal fraction measurements are shown for all subjects as a function of age for the first measurement.

In Table 3, linear regression slopes and confidence intervals are shown for both groups. For the control group, a significant decrease of WM fraction and brain 
Table 3: Linear regression of the normalized WMF, GMF, CSFF, NF, and BPF of the control and the MS groups as a function of age ${ }^{a}$

\begin{tabular}{lccccc}
\hline & WMF & GMF & CSFF & NF & BPF \\
\hline Contrl-age (\%/yr) & $-0.12(-0.21 \text { to }-0.03)^{\mathrm{b}}$ & $-0.03(-0.11-0.05)$ & $0.17(0.07-0.27)^{\mathrm{c}}$ & $-0.01(-0.03-0.01)$ & $-0.17(-0.27 \text { to }-0.07)^{\mathrm{c}}$ \\
Contr2-age (\%/yr) & $-0.10(-0.19 \text { to }-0.01)^{\mathrm{b}}$ & $-0.05(-0.12-0.03)$ & $0.17(0.07-0.27)^{\mathrm{c}}$ & $-0.02(-0.05-0.02)$ & $-0.17(-0.2 \text { to }-0.07)^{\mathrm{c}}$ \\
MS1-age (\%/yr) & $-0.02(-0.26-0.22)$ & $-0.03(-0.16-0.10)$ & $0.05(-0.13-0.23)$ & $0.00(-0.08-0.07)$ & $-0.05(-0.23,-0.13)$ \\
MS2-age (\%/yr) & $-0.01(-0.31-0.30)$ & $-0.05(-0.25-0.15)$ & $0.04(-0.14-0.23)$ & $0.02(-0.06-0.09)$ & $-0.04(-0.23-0.14)$ \\
MS1-EDSS (\%/unit) & $-1.16(-2.28 \text { to }-0.05)^{\mathrm{b}}$ & $-0.17(-0.87-0.54)$ & $0.93(-0.19-2.04)$ & $0.23(-0.14-0.62)$ & $-1.08(-1.90 \text { to }-0.26)^{\mathrm{b}}$ \\
MS2-EDSS (\%/unit) & $-1.34(-2.81-0.13)$ & $0.09(-1.01-1.19)$ & $0.93(-0.19-2.06)$ & $0.19(-0.20-0.58)$ & $-1.06(-1.92 \text { to }-0.20)^{\mathrm{b}}$ \\
\hline
\end{tabular}

Note:-Contr indicates control group; MS, MS patient group; 1, measurement 1; 2, measurement 2; WMF, WM fraction; GMF, GM fraction; CSFF, CSF fraction; NF, remaining, unclassified non-WM/GM/CSF tissue fraction; BPF, brain parenchymal fraction.

a The $95 \%$ confidence interval is given between parentheses. For the MS group, the linear regression of the age-corrected fractions with EDSS is provided.

${ }^{\mathrm{b}} P<.05$ (significant difference).

${ }^{c} P<.005$ (significant difference).
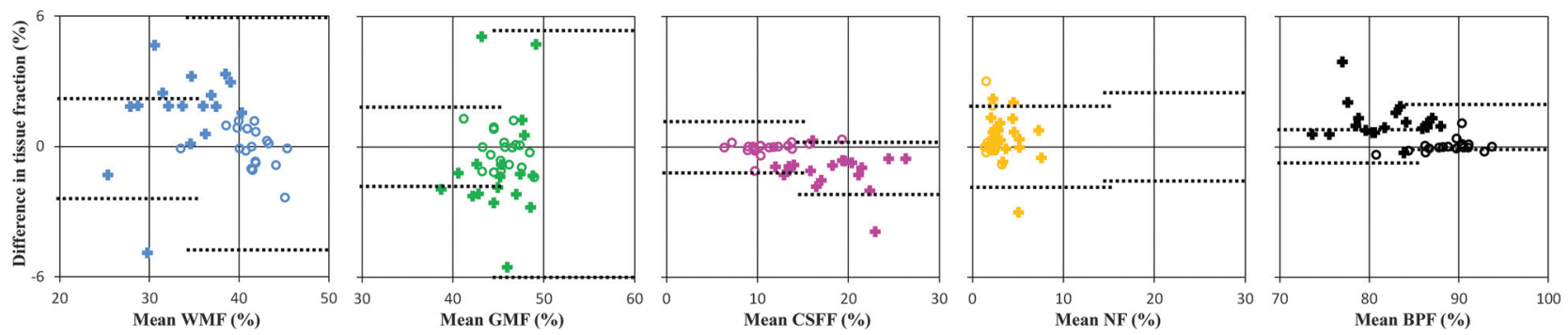

FIG 3. Bland-Altman plots for WM fraction, GM fraction, CSF fraction, remaining, unclassified non-WM/GM/CSF tissue fraction, and brain parenchymal fraction of all subjects with the mean tissue fraction against the difference in tissue fraction between measurements 1 and 2 . The dotted lines indicate the mean difference $\pm 2 \mathrm{SDs}$, on the left for the controls and on the right for the MS group. The scaling on both axes is identical; the colors are identical to those in Fig 2.

parenchymal fraction and a significant increase of CSF fraction with age were observed. No significant correlation with age was found for the MS group. After age correction, the patients with MS showed a significant decrease of brain parenchymal fraction and of WM fraction. Between measurements 1 and 2, the regression values were very similar and no significant difference was observed for any slope for either group. In Fig 3, Bland-Altman plots are shown for WM fraction, GM fraction, CSF fraction, remaining, unclassified non-WM/GM/CSF tissue fraction, and brain parenchymal fraction for all subjects, in which the mean tissue fraction was plotted against the difference in tissue fraction between measurements 1 and 2. The 2 times SD areas are indicated by the dotted lines for both groups, on the left for the controls, and on the right for the MS group. For WM fraction and GM fraction, the SD of the patient group is much larger than that for the control group; for CSF fraction, remaining, unclassified non-WM/GM/CSF tissue fraction, and brain parenchymal fraction, it was similar.

\section{DISCUSSION}

In this work, a recently developed method of combining quantitative MR imaging with fully automatic tissue classification, synthetic tissue-mapping, was used to investigate brain-tissue volumes of patients with MS pre- and post-contrast agent administration. This method is both fast and objective because brain segmentation can be obtained in a total time of 10 minutes (acquisition plus postprocessing) and without user interaction for tissue classification.

In a clinical and research context, accurate measurement of the ICV is important because it is used to normalize brain-tissue volumes to reduce the effect of differences in subject head size. ${ }^{31}$ The repeatability of the ICV segmentation was estimated by using the 2 measurements of the control group. The software reproduced values for the repeated measurements with a nonsignificant difference of $2 \mathrm{~mL}$, which corresponds to only $0.14 \%$ of the ICV. The intrasubject SD in this cohort was $0.50 \%$, which was somewhat lower than the $0.83 \%$, found in a previous assessment by Ambarki et al. ${ }^{24}$ The administration of Gd, however, had a significant effect on the measurement of the ICV; after contrast agent administration, the ICV was $13 \mathrm{~mL}$ smaller. Visual inspection did not result in locating a specific area where differences in ICV were pronounced, and ICV was measured slightly smaller on every slice. Our interpretation of these observations is that the slight reduction in the $\mathrm{T} 1$ relaxation of brain tissue due to the presence of Gd contrast agent in the capillary network resulted in a slight decrease of the measured PD, though PD should be invariant to T1. A lower PD will consequently shift the ICV border somewhat because the border was defined in the software at $\mathrm{PD}=50 \%$. A reduction of the ICV with a mean of $13 \mathrm{~mL}$, however, is relatively small. If one considers a $1387-\mathrm{mL}$ skull as a spheric object, the volume loss would correspond to a radial decrease of $0.2 \mathrm{~mm}$. Such a reduction is well below the resolution of our acquisitions.

The BPV was identical for both measurements and for both groups. The reduction of the ICV, observed post-Gd in patients with MS, resulted in a reduction of the observed CSFV. This means that the ratio between BPV and ICV, the brain parenchymal fraction, changed, as shown in Table 2, with a significant increase of $1.0 \%$ in a paired test. The brain parenchymal fraction is considered a valid measure for brain atrophy, ${ }^{32}$ and the effect of the presence of $\mathrm{Gd}$ is therefore important for follow-up. The apparent increase of brain parenchymal fraction of $1.0 \%$ was smaller than the observed variation of brain parenchymal fraction within the MS group (4.2\%). Furthermore, it was much smaller than the difference between the MS group and the controls (6.9\% for measurement 1 and $6.0 \%$ for measurement 2). Linear regression (Table 3 ) showed that the difference pre- and post-Gd appears to be 
an offset, which was similar for all subjects; the measured slopes as a function of age and EDSS were very similar for both measurements. The results of this study suggest that a common practice, comparing images of patients with MS acquired post-Gd with those of healthy controls without Gd should still be considered a valid approach, despite the effects of Gd on the tissue-classification volumes. Further investigation is required to confirm this for patients with MS at earlier stages of the disease and patients with MS with enhancing lesions.

The results for brain parenchymal fraction in this work were very close to the values of Vagberg et $\mathrm{al},{ }^{25}$ who reported a brain parenchymal fraction of $85.2 \%$ for the MS group on the basis of analysis using the same software. These patients had a median EDSS $=2.5$, which was acquired post-Gd. In this work, the mean (post-Gd) brain parenchymal fraction was $82.9 \%$ for patients, who had a higher median EDSS $=3.5$. For the control group, it was $89.0 \%$ (no Gd), compared with our $88.8 \%$. The coefficients of variation of repeated BPF measurements were comparable.

The measured values for brain parenchymal fraction for the control group were higher than those in a large study by DeCarli et $\mathrm{al}^{33}$ in which a mean brain parenchymal fraction of $78 \%$ was found (mean subject age, 62 years). Differences in absolute brain parenchymal fraction values may have various causes: DeCarli et al, for example, used another definition of the ICV, in which the cerebellum was excluded. Furthermore, CSF was manually segmented in a binary fashion by using an image-intensity threshold. Because any method has a cascade of segmentation process details, they easily lead to differences in the final results. As shown in our work, the absence or presence of Gd contributes to these differences. It is, therefore, clinically more relevant to focus on the change of tissue classification values with time or over disease severity. As shown in Table 3, there was no significant difference in the correlation of the volume fractions with age and EDSS. In the study of DeCarli et al, a decrease of brain parenchymal fraction of $0.26 \%$ per year was reported, an observation that was within the $95 \%$ confidence interval of our measurements. The lower rate of $0.17 \%$ that was found could partly be explained by the fact that our subjects were on average 14 years younger.

Gd contrast agent is expected to decrease the T1 relaxation in brain tissue. Even though no enhancing lesions were observed for the included patients with MS, a nonzero Gd concentration must have been present in the capillary network. Because of this shift toward shorter T1 relaxation times, the segmentation results may show an apparent increase of the brain tissue with the shortest $\mathrm{T} 1$ relaxation time (ie, white matter). As seen in Tables 1 and 2, there was a trend that WM increased somewhat, at the expense of GM post-Gd, but this effect was not significant. The Gd concentration in nonlesional normal-appearing brain tissue must, therefore, be relatively low.

Within the ICV, some tissue was not recognized as either WMV, GMV, or CSFV, and was labeled NV. The advantage of the presence of a fourth class for the remaining, unclassified tissue fraction is that it avoids forcing tissue into the WMV, GMV, or CSFV classes. Therefore, it reduces the effect of the presence of white matter lesions, which can be a problem for automatic braintissue-classification algorithms. ${ }^{34}$ As is shown in Fig 1, white matter lesions mainly show up in NV; therefore, it could serve as a basis for MS lesion-load estimation. An offset of the NV, corre- sponding to $28-31 \mathrm{~mL}(2.0 \%-2.2 \%$ of the ICV $)$, was observed in healthy subjects, mainly consisting of blood vessels and small rims at the intracranial edge. For the MS group, the NV was significantly higher, at $52 \mathrm{~mL}$ pre-Gd and $60 \mathrm{~mL}$ post-Gd $(3.8 \%$ and $4.4 \%$ of the ICV, respectively), suggesting that $24-29 \mathrm{~mL}$ might be assigned to MS lesion volume. Although these higher values suggests that the NV might be useful for MS lesion-load measurements, currently, extensive manual correction will be required, both to reduce false-positives of nonlesional areas and to correct false-negatives when MS lesions are recognized as partial volume GM or CSF instead. The effect of Gd was significant, apparently increasing the potential MS volume to $8 \mathrm{~mL}$ post-Gd, a $15 \%$ increase in comparison with the pre-Gd volume. Further investigation on, for example, the reproducibility and the influence of the rather thick sections $(4 \mathrm{~mm})$ is required to confirm this finding. It could be speculated that the introduction of a fifth class, blood vessels, could improve the specificity of the NV to segment MS lesions. Blood vessels typically have very low $\mathrm{PD}$ values because synthetic tissue-mapping uses a blackblood acquisition sequence.

A limitation of the study was the low number of included subjects and the combination of subjects diagnosed with relapsing-remitting MS and secondary-progressive MS in the group of patients with MS. The effects of Gd may, therefore, be different for the different MS groups. Furthermore, only single-dose Gd and a fixed time after administration were investigated. Although these 2 variables were carefully controlled, the absolute Gd concentration may still have varied over the patients due to, for example, weight, blood volume, and perfusion rate. As seen in Fig 3, the variation in segmentation results increased significantly for WM and GM, which may be a sign of different Gd concentrations. Administration of double-dose Gd, or choosing another delay time after administration of Gd, may further affect the automatic tissue classification. The results of the synthetic tissue-mapping method, however, are congruent with the results of other, larger studies on controls and patients with MS. A limitation for the repeated measurements was the lack of measurements for controls with Gd and patients with MS without Gd, which was beyond the scope of our ethical permission. Therefore, the observed effect of changes in the automatic segmentation after Gd administration may be specific for our groups. Especially, the effect of minor Gd leakage on the MS lesions can be of influence because the measured NV did increase to $8 \mathrm{~mL}$ post-Gd. It is more likely, however, that the increase of NV occurred simultaneously with a decrease of the sum of WMV and GMV (observed 12-17 = -5 $\mathrm{mL}$ ) and therefore did not have an influence on BPV. The effects on automatic tissue classification of other patient populations, other Gd concentrations, and acquisitions on other scanner brands are subject to future investigations of the method.

A potential limitation could be the relatively low resolution of the method, which could decrease the variation between measurements. The method, however, is relatively insensitive to resolution, owing to the incorporation of a partial volume model, and it has been shown that the volume measurements are similar by using a variety of resolutions. ${ }^{16}$ It is, therefore, not expected that the application of a higher spatial resolution would alter the differences pre- and post-Gd. The effect of choosing different geometries was not investigated.

AJNR Am J Neuroradiol 35:1330-36 Jul 2014 www.ajnr.org 1335 


\section{CONCLUSIONS}

The synthetic tissue-mapping method provides automatic measurements of WMV, GMV, CSFV, NV, BPV, and ICV, with a high degree of repeatability within a short time. The administration of Gd contrast agent in patients with MS had a significant effect on the tissue-classification results, and changes were observed for CSFV, NV, and ICV. For brain-tissue fractions, normalized with the ICV, changes were observed in CSF fraction, remaining, unclassified non-WM/GM/CSF tissue fraction, and brain parenchymal fraction. The observed changes in this study, however, were much smaller than the differences between the group of patients with MS and healthy controls. There were no significant differences between the correlations with age and EDSS pre- and post-Gd.

Disclosures: J.B.M. Warntjes—UNRELATED: Employment: Synthetic MR AB, Comments: I am employed part-time at Synthetic MR AB, a software company specializing in MR image analysis and the manufacturer of SyMRI 7.0, Stock/Stock Options: Synthetic MR AB, Comments: I own shares. P. Lundberg—RELATED: Grant: National Research Council/NT*, UNRELATED: Board Membership: Amra AB, Comments: not related to this work (no money paid), Grants/Grants Pending: National Medical Research Council,* VINNOVA, * Comments: funding for other research, Patents (planned, pending or issued): Improvement in Magnetic Resonance Imaging Relating to Correction of Chemical Shift Artifact and Intensity Inhomogeneity, EP Patent 2,283,376; Improvement in Magnetic Resonance Imaging Relating to Correction of Chemical Shift Artifact and Intensity Inhomogeneity, US Patent 20, 110,091,090, Comments: held by Amra AB (no money received), Stock/Stock Options: Amra AB, Comments: no money paid, Travel/Accommodations/Meeting Expenses Unrelated to Activities Listed: paid by my employer (County Council University Hospital) for attending ISMRM. *Money paid to the institution.

\section{REFERENCES}

1. Barkhof F. The clinico-radiological paradox in multiple sclerosis revisited. Curr Opin Neurol 2002;15:239-45

2. Chard D, Miller D. Is multiple sclerosis a generalized disease of the central nervous system? An MRI perspective. Curr Opin Neurol 2009;22:214-18

3. Minneboo A, Uitdehaag BM, Jongen P, et al. Association between MRI parameters and the MS severity scale: a 12 year follow-up study. Mult Scler 2009;15:632-37

4. Zivadinov R, Cox JL. Neuroimaging in multiple sclerosis. Int Rev Neurobiol 2007;79:449-74

5. Filippi M, Tortorella C, Bozzali M. Normal-appearing white matter changes in multiple sclerosis: the contribution of magnetic resonance techniques. Mult Scler 1999;5:273-82

6. Miller DH, Barkhof F, Frank JA, et al. Measurement of atrophy in multiple sclerosis: pathological basis, methodological aspects and clinical relevance. Brain 2002;125:1676-95

7. Mortazavi D, Kouzani AZ, Soltanian-Zadeh H. Segmentation of multiple sclerosis lesions in MR images: a review. Neuroradiology 2012;54:299-320

8. Grassiot B, Desgranges B, Eustache F, et al. Quantification and clinical relevance of brain atrophy in multiple sclerosis: a review. J Neurol 2009;256:1397-412

9. Chard DT, Griffin CM, Parker GJ, et al. Brain atrophy in clinically early relapsing-remitting multiple sclerosis. Brain 2002;125:327-37

10. Andersen AH, Zhang Z, Avison MJ, et al. Automated segmentation of multispectral brain MR images. J Neurosci Methods 2002; 122:13-23

11. Alfano B, Brunetti A, Covelli EM, et al. Unsupervised, automated segmentation of the normal brain using a multispectral relaxometric magnetic resonance approach. Magn Reson Med 1997;37:84-93

12. Ishii K, Soma $\mathrm{T}$, Kono AK, et al. Automatic volumetric measurement of segmented brain structures on magnetic resonance imaging. $\mathrm{Ra}$ diat Med 2006;24:422-30

13. Ashburner J, Friston KJ. Unified segmentation. Neuroimage 2005; 26:839-51
14. Fischl B. FreeSurfer. Neuroimage 2012;62:774-81

15. Hasan KM, Walimuni IS, Abid $\mathrm{H}$, et al. Human brain atlas-based multimodal MRI analysis of volumetry, diffusimetry, relaxometry and lesion distribution in multiple sclerosis patients and healthy adult controls: implications for understanding the pathogenesis of multiple sclerosis and consolidation of quantitative MRI results in MS. J Neurol Sci 2012;313:99-109

16. West J, Warntjes JB, Lundberg P. Novel whole brain segmentation and volume estimation using quantitative MRI. Eur Radiol 2012; 22:998-1007

17. Anbeek P, Vincken KL, van Bochove GS, et al. Probabilistic segmentation of brain tissue in MR imaging. Neuroimage 2005;27:795-804

18. Shattuck DW, Sandor-Leahy SR, Schaper KA, et al. Magnetic resonance image tissue classification using a partial volume model. Neuroimage 2001;13:856-76

19. Kovacevic N, Lobaugh NJ, Bronskill MJ, et al. A robust method for extraction and automatic segmentation of brain images. Neuroimage 2002;17:1087-100

20. Jovicich J, Czanner S, Han X, et al. MRI-derived measurements of human subcortical, ventricular and intracranial brain volumes: reliability effects of scan sessions, acquisition sequences, data analyses, scanner upgrade, scanner vendors and field strengths. Neuroimage 2009;46:177-92

21. MacKay A, Laule C, Vavasour I., et al. Insights into brain microstructure from the T2 distribution. Magn Reson Imaging 2006;24: $515-25$

22. Larsson HB, Frederiksen J, Petersen, et al. Assessment of demyelineation, edema and gliosis by in-vivo determination of $\mathrm{T} 1$ and $\mathrm{T} 2 \mathrm{in}$ the brain of patients with acute attack of multiple sclerosis. J Magn Reson Med 1989;11:337-38

23. Pengas G, Pereira JMS, Williams GB, et al. Comparative reliability of total intracranial volume estimation methods and the influence of atrophy in a longitudinal semantic dementia cohort. J Neuroimaging 2009;19:37-46

24. Ambarki K, Lindqvist T, Wåhlin A, et al. Evaluation of automatic measurement of the intracranial volume based on quantitative MR imaging. AJNR Am J Neuroradiol 2012;33:1951-56

25. Vågberg M, Lindqvist T, Ambarki K, et al. Automated determination of brain parenchymal fraction in multiple sclerosis. AJNR Am J Neuroradiol 2013;34:498-504

26. Warntjes JB, Dahlqvist Leinhard O, West J, et al. Optimization for clinical usage of rapid magnetic resonance quantification on the brain. Magn Reson Med 2008;60:320-29

27. Tofts P. Quantitative MRI of the Brain. Chichester, UK: Wiley; 2003

28. Poser CM, Paty DW, Scheinberg L, et al. New diagnostic criteria for multiple sclerosis: guidelines for research protocols. Ann Neurol 1983;13:227-31

29. McDonald WI, Compston A, Edan G, et al. Recommended diagnostic criteria for multiple sclerosis: guidelines from the International Panel on the Diagnosis of Multiple Sclerosis. Ann Neurol 2001;50: $121-27$

30. Kurtzke JF. Rating neurologic impairment in multiple sclerosis: an expanded disability status scale (EDSS). Neurology 1983;33:1444-52

31. Whitwell JL, Crum WR, Watt HC, et al. Normalization of cerebral volumes by use of intracranial volume: implications for longitudinal quantitative MR imaging. AJNR Am J Neuroradiol 2001;22: 1483-89

32. Rudick RA, Fisher E, Lee JC, et al. Use of brain parenchymal fraction to measure whole brain atrophy in relapsing-remitting MS: Multiple Sclerosis Collaborative Group. Neurology 1999;53:1698-704

33. DeCarli C, Massaro J, Harvey D, et al. Measures of brain morphology and infarction in the Framingham Heart Study: establishing what is normal. Neurobiol Aging 2005;26:491-510

34. Battaglini M, Jenkinson M, De Stefano N. Evaluating and reducing the impact of white matter lesions on brain volume measurements. Hum Brain Mapp 2012;33:2062-71 